\title{
Thromboelastography Platelet Mapping-A Useful Preoperative Tool to Reduce Transfusion Requirement by Determining Timing of Coronary Artery Bypass Graft Surgery in Patients taking Dual Antiplatelet Medication: A Pilot Study from Eastern India
}

\author{
Suvro Sankha Datta ${ }^{1, \oplus}$ Dibyendu De $^{2}$ Satyajit Bose ${ }^{3}$ \\ ${ }^{1}$ Department of Transfusion Medicine, The Mission Hospital, \\ Durgapur, West Bengal, India \\ 2Department of Clinical Hematology, The Mission Hospital, \\ Durgapur, West Bengal, India \\ ${ }^{3}$ Department of Cardio-Vascular Surgery, The Mission Hospital, \\ Durgapur, West Bengal, India
}

J Card Crit Care:2021;5:23-28

\author{
Address for correspondence Suvro Sankha Datta, MD, Department \\ of Transfusion Medicine, The Mission Hospital, Durgapur, West \\ Bengal, 713212, India (e-mail: suvro.datta@gmail.com). \\ Present address Department of Transfusion Medicine, Tata Medical \\ Center, Newtown, Rajarhat, 700160, Kolkata, India.
}

\begin{abstract}
Keywords

- thromboelastography

- platelet-mapping

- DAPT

- CABG

- transfusion

- timing of surgery

In this pilot study, we proposed an algorithm to use thromboelastography platelet mapping (TEG-PM) as an effective preoperative platelet function assessment tool in coronary artery bypass graft surgery (CABG) to reduce the transfusion requirement by determining timing of surgery in patients who are on dual antiplatelet therapy (DAPT). Total 15 elective CABG patients who were receiving DAPT were tested by TEG-PM assay after 72 hours' stoppage of both medications. Four patients who had $<50 \%$ inhibition with agonists (arachidonic acid [AA] and adenosine diphosphate [ADP]) were operated within 24 hours of assay. Eight patients who had $>70 \%$ inhibition with AA/ADP, surgery was deterred till 48 hours, and 3 patients with $>50 \%$ but $<70 \%$ inhibition were operated within 24 hours after preoperative prophylactic platelet transfusion. Transfusion requirement was recorded and compared with the previous transfusion data for CABG cases operated without TEG-PM. Distribution of ADP inhibition was $67.54 \pm 30.03 \%$ and $A A$ inhibition was $60.69 \pm 30.14 \%$, showing a variable offset of the effect of both medications starting after 72 hours of cessation. Overall transfusion requirement was decreased by using TEG-PM algorithm. Preoperative platelet function testing to determine the timing of surgery by TEG-PM assay in DAPT-treated patients may be a useful strategy to reduce transfusion requirement in CABG.
\end{abstract}

\section{Introduction}

Dual antiplatelet therapy (DAPT) with acetylsalicylic acid and a P2Y12 receptor antagonist reduces the risk of thrombosis after percutaneous coronary intervention $(\mathrm{PCI})$ in

published online January 22, 2021
DOI https://doi.org/ $10.1055 / \mathrm{s}-0041-1723622$ ISSN 2457-0206. acute coronary syndrome cases. ${ }^{1}$ Irreversible inhibition of platelet function associated with DAPT carries a substantial risk of bleeding, particularly in patients undergoing coronary artery bypass graft surgery $(\mathrm{CABG}){ }^{2}$ In addition to that

\footnotetext{
(C) 2021. Official Publication of The Simulation Society (TSS), accredited by International Society of Cardiovascular Ultrasound (ISCU).

This is an open access article published by Thieme under the terms of the Creative Commons Attribution-NonDerivative-NonCommercial-License, permitting copying and reproduction so long as the original work is given appropriate credit. Contents may not be used for commercial purposes, or adapted, remixed, transformed or built upon. (https://creativecommons.org/licenses/by-nc-nd/4.0/) Thieme Medical and Scientific Publishers Pvt. Ltd., A-12, 2nd Floor, Sector 2, Noida-201301 UP, India
} 
platelet dysfunction is a well-known side effect of coronary artery bypass and has a significant impact on blood transfusion policy in CABG. ${ }^{3}$ Current international guidelines recommend discontinuation of the P2Y12 receptor antagonist at least 5 days before cardiac surgery. ${ }^{4}$ However, a significant number of patients still undergo CABG after a shorter discontinuation time despite an increase bleeding risk. Currently, the transfusion policy of blood and blood components to manage bleeding in CABG cases is largely empirical in India and widely varied among institutions. In 2011, the Society of Thoracic Surgeons and the Society of Cardiovascular Anaesthesiologists' Blood Conservation Clinical Practice Guidelines gave a recommendation for platelet function testing to determine the timing of CABG in patients on DAPT (Class IIb, Level of Evidence C). ${ }^{5}$ Simple and rapid methodologies to evaluate platelet function in patients on DAPT might be useful for clinical practice. Thromboelastography platelet mapping (TEG-PM) is an example of one such platelet function test which could be effectively used to predict blood loss in CABG cases by detecting alterations in platelet receptor activity before or after surgery. ${ }^{6}$ There have been conflicting recommendations for prophylactic platelet transfusion in CABG. Although, the Society of Thoracic Surgeons advises for platelet transfusion in high-risk cardiac patients for bleeding prevention, ${ }^{5}$ according to the British Society of Haematology, ${ }^{7}$ the role of prophylactic platelet transfusion to reverse the effect of antiplatelet therapy is still not clear.

In this pilot study, we proposed an algorithm based on platelet function assessment in CABG cases and evaluated whether TEG-PM assay could be effectively used as a preoperative tool to reduce the transfusion requirement by determining timing of surgery in patients who were on DAPT.

\section{Materials and Methods}

\section{Study Details}

It was a single-center observational pilot study conducted in adult patients who were on DAPT and underwent CABG. Prospective data were collected on 15 patients who were admitted for CABG from December 2017 onwards. The study was approved by the local ethics committee and informed consent was obtained from each patient.

\section{Study Population}

All patients who were admitted for elective CABG and had $\mathrm{PCI}$ done within 1 year of admission date were included in this study. Each patient was receiving aspirin and clopidogrel in combination and implanted with a drug-eluting coronary stent. The exclusion criteria were re-exploration surgeries, CABG in combination with valve surgery, off-pump surgery, emergency surgery, preexisting bleeding diathesis, active liver disease, platelet count less than $100 \times 10^{9} / \mathrm{L}$ or greater than $500 \times 10^{9} / \mathrm{L}$, prothrombin time $(\mathrm{PT})>1.5$ times of control, hematocrit $<30 \%$, creatinine $>0.354 \mathrm{mmol} / \mathrm{L}$, any use of other anticoagulatory medication apart from aspirin and clopidogrel, or any recent utilization of nonsteroidal anti-inflammatory drugs. All patients were receiving a standard dose of DAPT according to their body weight before preoperative cessation of medications and on-pump surgeries were performed by the same team of cardiac surgeons and anesthesiologists. In the operation theater, a standard preoperative, anesthetic, surgical, and postoperative treatment was provided to all patients according to the institutional protocol.

\section{Blood Sampling and Platelet Function Analysis}

Blood samples were drawn from each patient after 72 hours' stoppage of both antiplatelet medications and transferred to separate vacutainer blood collecting tubes (BD Vacutainer, Becton Dickinson, United Sates) containing 3.2\% trisodium citrate or lithium heparin. After discarding the first 2 to $3 \mathrm{~mL}$ of blood, the vacutainer tubes were filled to the capacity and gently inverted three to five times to ensure complete mixing of the anticoagulant. After collection samples were immediately sent to the transfusion medicine department for TEG-PM analysis. Blood was analyzed by trained personnel within an hour of receiving the blood sample as per the manufacturer's instructions (Haemoscope Corporation; TEG Guide to Platelet Mapping; Monitor anti-platelet therapy; 2008). ${ }^{8}$ Both analyzer (series 5000) and the reagents were from Haemoscope Corporation (Niles, Illinois, United States). TEG parameters studied were reaction time $(R)$ : time to initial fibrin formation up to $2 \mathrm{~mm}$, $\mathrm{K}$ time $(K)$ : time to clot formation up to $20 \mathrm{~mm}, \alpha$ angle $(\alpha)$ : speed of clot formation and maximum amplitude (MA): measurement of clot strength. The normal reference values of TEG parameters (as provided by the manufacturer) for kaolin-activated citrated samples were taken as $R=2$ to 8 minutes, $K=1$ to 3 minutes, $\alpha=55$ to 78 degrees, and MA = 51 to $69 \mathrm{~mm}$. Heparin was used as an anticoagulant to eliminate thrombin activity in the sample during assay. Reptilase and activated factor XIII (activator F) were used to generate a cross-linked fibrin clot to isolate the fibrin contribution in clot strength. ${ }^{9}$ For maximal clot strength $\left(\mathrm{MA}_{\text {Thrombin }}\right) 1 \mathrm{~mL}$ of citrate stabilized blood was transferred to a vial containing kaolin and mixed five times by gentle inversion. Kaolin-activated blood (340 $\mu \mathrm{L}$ ) was added to a TEG cup containing $20 \mu \mathrm{L}$ of $0.2 \mathrm{M} \mathrm{CaCl}_{2}$. As MA, which is representing the maximal clot strength, can be ascertained by the binding of activated platelets to a fibrin mesh, $360 \mu \mathrm{L}$ of heparinized blood was added to $10 \mu \mathrm{L}$ of activator $\mathrm{F}$ (reptilase and factor XIIIa) and the contribution of each fibrin meshwork to clot strength $\left(\mathrm{MA}_{\text {Fibrin }}\right)$ was assessed. The contribution of P2Y12 receptor or cyclooxygenase pathways to the clot formation can be measured by the addition of the appropriate agonist, adenosine diphosphate (ADP) or arachidonic acid (AA). Therefore, ADP and AA, respectively, were added to activator $F$ to measure the degree of ADP receptor and thromboxane A2 (TXA2)-induced platelet aggregation. ${ }^{9}$ Heparinized blood $(360 \mu \mathrm{L})$ was added to a TEG cup in the presence of the activator F and agonist, $10 \mu \mathrm{L}$ $\operatorname{ADP}(2 \mu \mathrm{M}$, final concentration $)$ yielding the $\mathrm{MA}_{\mathrm{ADP}}$ or $10 \mu \mathrm{L}$ AA ( $1 \mu \mathrm{M}$, final concentration) for the $\mathrm{MA}_{\mathrm{AA}}$. Percentage of platelet inhibition was defined by the extent of nonresponse of the platelet ADP or TXA2 receptor to the exogenous ADP and AA as measured by $\mathrm{TEG}_{\mathrm{MA}}$. The percentage of platelet aggregation to agonist was calculated by: $\left[\left(\mathrm{MA}_{\mathrm{ADP} / \mathrm{AA}}-\mathrm{MA}_{\mathrm{Fibrin}}\right) /\right.$ 
$\left.\left(\mathrm{MA}_{\text {Thrombin }}-\mathrm{MA}_{\text {Fibrin }}\right) \times 100\right]$ and \% inhibition $=(100 \%-\%$ aggre gation). The calculation was performed by the TEG-PM software to get the percentage of platelet inhibition in patients as shown in - Fig. 1.

\section{Study Algorithm}

The TEG-PM-based algorithm for this study is presented in -Fig. 2. Eligible patients were categorized into three groups depending on the percentage of inhibition to agonists. Patients who had $<50 \%$ inhibition with agonists were operated within 24 hours of assay. Patients with $>70 \%$ inhibition with AA or ADP, surgery was deterred till 48 hours, and patients who had $>50 \%$ but $<70 \%$ inhibition to any of the agonists were operated within 24 hours after preoperative prophylactic platelet transfusion. Perioperative and 24-hour postoperative transfusion requirement was recorded as endpoints in each group and compared with the previous institutional transfusion data for CABG cases operated without TEG. ${ }^{10}$ Preoperative variables, demographic details of patients, total duration of surgery, assessment of coagulopathy, and transfusion thresholds were similar to the data reported previously. ${ }^{10}$

\section{Data Collection and Statistical Analysis}

Data were recorded and analyzed by using Microsoft Excel statistics software package. Continuous variables were expressed as mean \pm standard deviation and median with interquartile range. Student's $t$-test was applied for

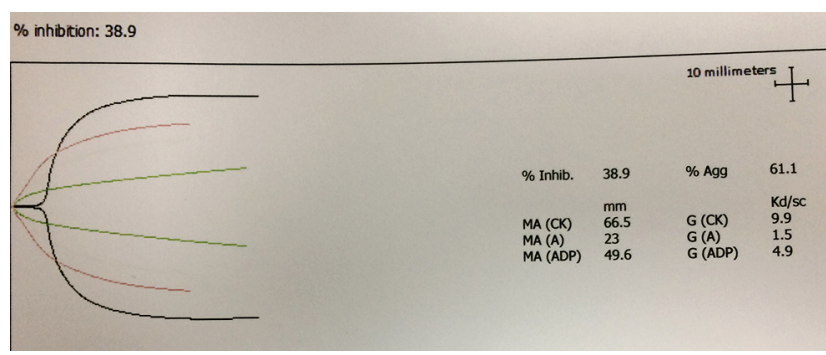

Fig. 1 Pre-operative thromboelastography platelet mapping (TEG-PM) tracing. comparison of means and significance was established with $p$-values of $<0.05$.

\section{Results}

-Table 1 summarizes all details regarding patients, tests, time of surgery, and transfusion records. Distribution of ADP inhibition was $67.54 \pm 30.03 \%$ and AA inhibition was $60.69 \pm$ $30.14 \%$ (-Table 2). Fig. 2 shows the number of patients operated under each group. Patients operated with TEG-PM assay showed a significant decrease in utilization of blood components like packed red blood cell (1.73 vs. $3.5 \mathrm{U} ; p<0.05)$ and fresh frozen plasma (1.6 vs. $3.8 \mathrm{U} ; p<0.05$ ) when compared with the institutional historical cohort data for similar group of patients operated in absence of TEG..$^{10}$ Platelet transfusion (four units random donor platelets) was needed only for patients categorized under the prophylactic group.

\section{Discussion}

Timing of elective cardiac surgery is a major concern in patients with coronary artery stents who are receiving DAPT. The risk of excessive bleeding must be carefully balanced against the increased risk that discontinuing antiplatelet therapy before CABG has on thrombotic events. ${ }^{11}$ This study has demonstrated that patients who have recently suspended DAPT for surgery have variable levels of preoperative platelet inhibition. This phenomenon might be due to the interindividual variability of platelet response. Because there is wide variability in response and also variability in platelet function recovery especially after clopidogrel withdrawal, a uniform waiting period may not benefit all patients needing CABG as concluded by Collyer et al. ${ }^{12}$ Preisman et al ${ }^{13}$ also comment on this lack of patient responsiveness to therapy by stressing the importance of measuring platelet function in patients undergoing cardiothoracic surgery to reduce postoperative blood loss. A recent modification of the TEG analysis that includes PM has generated interest as a platelet function test. Inconsistencies have been noticed between the ADP or the AA pathways measured

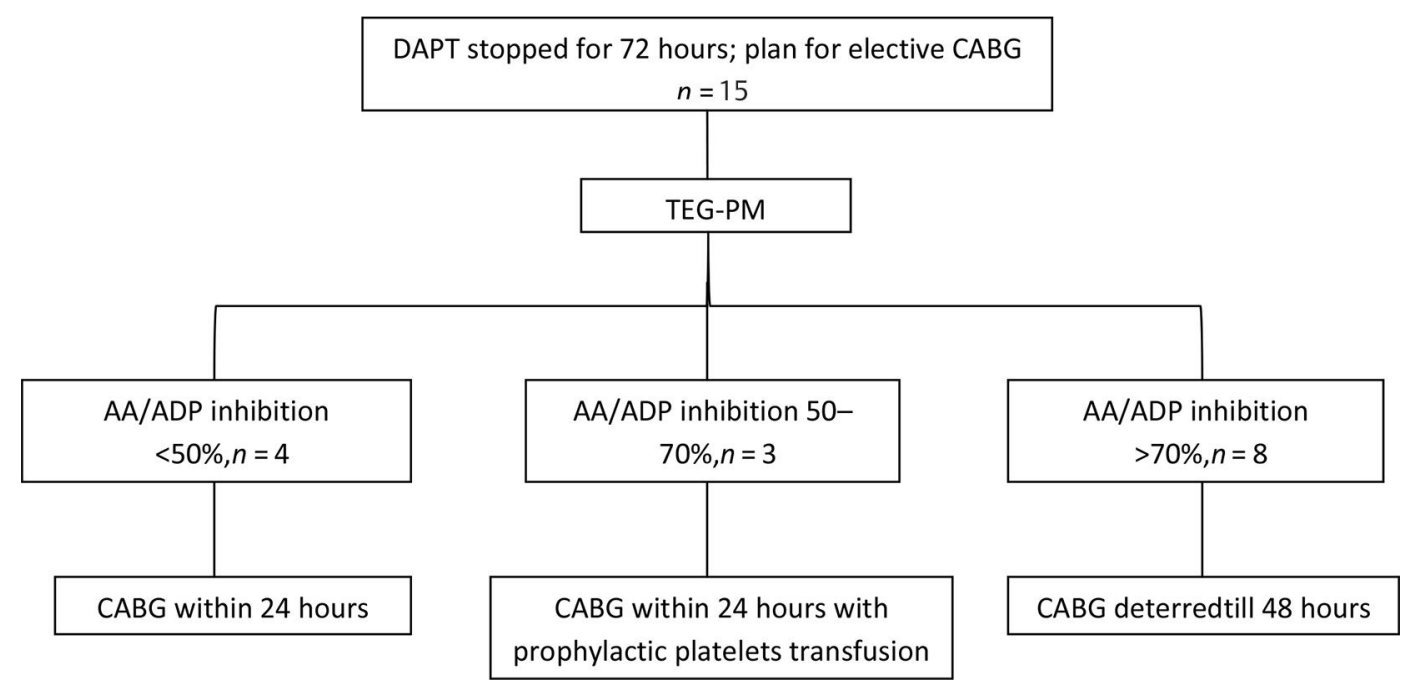

Fig. 2 Thromboelastography platelet mapping (TEG-PM)-based algorithm for cardiac surgery. 
Table 1 Details of patients, tests, time of surgery, and transfusion records

\begin{tabular}{|c|c|c|c|c|c|c|c|c|c|c|c|c|}
\hline \multirow[t]{2}{*}{ SL. } & \multirow[t]{2}{*}{ Age/Sex } & \multirow{2}{*}{$\begin{array}{l}\text { Platelet } \\
\text { counts }\end{array}$} & \multicolumn{8}{|c|}{ TEG-PM parameters } & \multirow{2}{*}{$\begin{array}{l}\text { Time of } \\
\text { surgery } \\
\text { after } \\
\text { TEG-PM }\end{array}$} & \multirow[t]{2}{*}{ Transfusion records } \\
\hline & & & $R$ & $K$ & Alpha & $\mathrm{MA}_{\mathrm{CK}}$ & $\mathrm{MA}_{\mathrm{AA}}$ & $M A_{A D P}$ & $\begin{array}{l}\text { AA } \\
\text { inh }\end{array}$ & $\begin{array}{l}\text { ADP } \\
\text { inh }\end{array}$ & & \\
\hline 1 & $64 / \mathrm{M}$ & $158 \times 10^{9} / \mathrm{L}$ & 6.2 & 2.0 & 63.6 & 61.3 & 10.1 & 3.1 & 99.2 & 100 & After $48 \mathrm{~h}$ & PRBC: 2; FFP: 2 \\
\hline 2 & $64 / M$ & $175 \times 10^{9} / \mathrm{L}$ & 3.8 & 1.0 & 75.4 & 80.9 & 19.4 & 23.1 & 91.9 & 86.4 & After $48 \mathrm{~h}$ & PRBC: 2; FFP: 2 \\
\hline 3 & $65 / M$ & $168 \times 10^{9} / \mathrm{L}$ & 3.4 & 0.9 & 76.1 & 79.8 & 26.2 & 24.5 & 91.5 & 94.4 & After $48 \mathrm{~h}$ & PRBC: 2; FFP: 2 \\
\hline 4 & $68 / \mathrm{F}$ & $160 \times 10^{9} / \mathrm{L}$ & 8.2 & 4.5 & 40.8 & 65.0 & 18.3 & 13.5 & 83.4 & 92 & After $48 \mathrm{~h}$ & PRBC: 2; FFP: 3; CRYO: 5 \\
\hline 5 & $64 / M$ & $170 \times 10^{9} / \mathrm{L}$ & 3.8 & 1.2 & 75.5 & 76.0 & 63.1 & 47.1 & 20 & 44.5 & Within $24 \mathrm{~h}$ & PRBC: 2 \\
\hline 6 & $65 / M$ & $165 \times 10^{9} / \mathrm{L}$ & 4.3 & 1.3 & 70.3 & 67.6 & 54.1 & 57.4 & 24 & 18.1 & Within $24 \mathrm{~h}$ & PRBC: 1 \\
\hline 7 & $70 / F$ & $172 \times 10^{9} / \mathrm{L}$ & 4.2 & 1.1 & 74.2 & 77.5 & 6.4 & 19.8 & 100 & 86.2 & After $48 \mathrm{~h}$ & PRBC: 1 \\
\hline 8 & $67 / M$ & $165 \times 10^{9} / \mathrm{L}$ & 4.9 & 1.3 & 52.3 & 66.5 & 39.5 & 49.6 & 62.1 & 38.9 & Within $24 \mathrm{~h}$ & $\begin{array}{l}\text { PRBC: } 1 \text {; FFP: } 2 \\
\text { RDP: } 4\end{array}$ \\
\hline 9 & $34 / \mathrm{M}$ & $173 \times 10^{9} / \mathrm{L}$ & 2.3 & 1.0 & 75.0 & 78.5 & 57.7 & 17.7 & 31.3 & 91.4 & After $48 \mathrm{~h}$ & PRBC: 2 \\
\hline 10 & $45 / M$ & $170 \times 10^{9} / \mathrm{L}$ & 7.0 & 1.9 & 62.7 & 73.9 & 52.3 & 61.2 & 38.9 & 22.9 & Within $24 \mathrm{~h}$ & PRBC: 1; FFP: 1 \\
\hline 11 & $58 / \mathrm{M}$ & $178 \times 10^{9} / \mathrm{L}$ & 7.8 & 2.3 & 60.1 & 82.1 & 54.3 & 48 & 49.6 & 60.8 & Within $24 \mathrm{~h}$ & $\begin{array}{l}\text { PRBC: } 2 ; \\
\text { FFP: 3; RDP: } 4\end{array}$ \\
\hline 12 & $70 / \mathrm{M}$ & $166 \times 10^{9} / \mathrm{L}$ & 5.2 & 1.8 & 52.1 & 68.1 & 46.5 & 47.1 & 36.4 & 38.8 & Within $24 \mathrm{~h}$ & PRBC: 2; FFP: 2 \\
\hline 13 & $62 / \mathrm{M}$ & $168 \times 10^{9} / \mathrm{L}$ & 3.0 & 1.0 & 74.2 & 69.4 & 16.0 & 17.4 & 96.9 & 94.4 & After $48 \mathrm{~h}$ & $\begin{array}{l}\text { PRBC: 2; FFP: 1; } \\
\text { CRYO: } 5\end{array}$ \\
\hline 14 & $56 / \mathrm{M}$ & $160 \times 10^{9} / \mathrm{L}$ & 8.6 & 3.2 & 48.8 & 75.7 & 38.5 & 47.8 & 53.4 & 44.3 & Within $24 \mathrm{~h}$ & $\begin{array}{l}\text { PRBC: } 2 ; \\
\text { FFP: } 3 \text {; RDP: } 4\end{array}$ \\
\hline 15 & $66 / M$ & $152 \times 10^{9} / \mathrm{L}$ & 4.8 & 1.3 & 50.9 & 56.2 & 40.2 & 5.7 & 31.8 & 100 & After $48 \mathrm{~h}$ & PRBC: 2; FFP: 3 \\
\hline
\end{tabular}

Abbreviations: AA inh, arachidonic acid inhibition; ADP inh, adenosine diphosphate inhibition; CRYO, cryoprecipitate; F, female; FFP, fresh frozen plasma; M, male; MA, maximum amplitude; PRBC, packed red blood cells; RDP, random donor platelet; TEG-PM, thromboelastography platelet mapping. Note: Transfusion record includes perioperative and 24 hours postoperative duration.

Table 2 Summary of all parameters and transfusion details

\begin{tabular}{|l|l|l|l|}
\hline & Mean \pm standard deviation & Median & IQR (Q1-Q3) \\
\hline Age & $61.2 \pm 9.82$ & 64 & $60-66.5$ \\
\hline CPB time (min) & $132 \pm 23.6$ & 135 & $115.4-149.6$ \\
\hline Cross-clamp time (min) & $100 \pm 13.6$ & 102 & $92.2-107.8$ \\
\hline Baseline $R$ (min) & $5.17 \pm 1.96$ & 4.8 & $3.8-6.6$ \\
\hline Baseline $K$ (min) & $1.72 \pm 0.99$ & 1.3 & $1.05-1.95$ \\
\hline Baseline alpha (degrees) & $63.47 \pm 11.98$ & 63.6 & $52.2-74.6$ \\
\hline Baseline MA (MA ${ }_{\text {KK }}$ in mm) & $71.9 \pm 7.71$ & 73.9 & $67.05-78$ \\
\hline AA (MA ${ }_{A A}$ in mm) & $36.17 \pm 18.73$ & 39.5 & $18.85-53.2$ \\
\hline \% of inhibition AA & $60.69 \pm 30.14$ & 53.4 & $34.1-91.7$ \\
\hline ADP (MA ${ }_{\text {ADP }}$ in mm) & $32.2 \pm 19.53$ & 24.5 & $17.55-47.9$ \\
\hline$\%$ of inhibition ADP & $67.54 \pm 30.03$ & 86.2 & $41.6-93.2$ \\
\hline PRBC transfusion & $1.73 \pm 0.46$ & 2 & $1.5-2$ \\
\hline FFP transfusion & $1.6 \pm 1.18$ & 2 & $0.5-2.5$ \\
\hline Abbrions: AA, acis & & 2 \\
\hline
\end{tabular}

Abbreviations: AA, arachidonic acid; ADP, adenosine diphosphate; FFP, fresh frozen plasma; IQR, interquartile range; MA, maximal amplitude; PRBC, packed red blood cells; TEG, thromboelastography.

Note: $R, K$, and alpha $(\alpha)$ are standard TEG parameters.

by different point-of-care devices with evidence of lower sensitivity of TEG-PM assay. ${ }^{14,15}$ However, TEG-PM assay was retested in healthy individuals ${ }^{16}$ as well as in cardiac surgery ${ }^{13,17}$ and found to be adequately sensitive for platelet function assessment. No consensus has been reached regarding a validated cutoff for TEG-PM assay which could predict CABG-related bleeding. The rationality for scheduling cardiac surgery in patients depending on percentage of inhibition in this study was based on the evidence of published data ${ }^{12,13,17,18}$ which showed how cutoff values could be different depending on the several factors in patients undergoing CABG on DAPT. Deterring surgery for 48 hours 
in patients who had $>70 \%$ inhibition in our study was based on the results published by Kwak et al ${ }^{17}$ where irrespective of the time between drug withdrawal and surgery, patients in the highest group of platelet inhibitory response $>76.5 \%$ inhibition) had higher chest tube output and higher transfusion rates as compared with patients in the other groups. The protocol of prophylactic platelet transfusion in patients who had $>50 \%$ but $<70 \%$ inhibition and operated within 24 hours of assay is supported by the data published by Charif et $\mathrm{al}^{19}$ where it has been concluded that emergency CABG on DAPT is most likely feasible and safe, especially when patients receive prophylactic peroperative platelet transfusion. The results of our study showed that hemostatic algorithm-guided transfusion protocol could reduce the use of blood and blood component therapy in cardiac surgery which is in agreement with the conclusion made by Karanjkar et al. ${ }^{20}$ To the best of our knowledge, this is the first pilot study from India regarding platelet function measurement-based strategy to reduce transfusion requirement and optimize waiting period in DAPT-treated patients undergoing on-pump CABG. Our data also demonstrated that the strategy of platelet function measurement by TEG-PM shortened the waiting period (mean 3.7 vs. 5 days per patient) in $46.67 \%$ ( 7 out of 15 ) patients of our study cohort without increasing the total uses of blood component.

There are several limitations in this brief study including the small sample size. Single-center, nonrandomized study design could compromise the power of the results. Another limitation of this study was the inconsistent availability of other coagulation tests (activated partial thromboplastin time, PT, international normalized ratio) in our patient population. However, a baseline kaolin TEG was recorded for each patient and was considered a comprehensive test for the purposes of this study. The cost of the assay could also be considered a constraint for using this test routinely in lowand middle-income countries.

Finally, we conclude that preoperative platelet function testing to determine the timing of surgery by TEG-PM assay in DAPT-treated patients may be a useful targeted therapeutic strategy to reduce transfusion requirement in CABG cases. Although further research is needed to reconsider the definition of what constitutes appropriate cutoff values for TEG-PM in patients undergoing CABG on DAPT.

\section{Funding}

We did not receive any specific grant from funding agencies in the public, commercial, or not-for-profit sectors for this study.

\section{Ethics Approval}

Ethical approval was obtained from the Institution Ethics Committee of The Mission Hospital [ECR/587/Inst/ WB/2014/RR-17] for this study.

\section{Consent}

Individual informed written consent was obtained from the patients before surgery.

\section{Data Transparency}

Raw data are available; data sheet can be shared on request.

\section{Authors' Contributions}

S.S.D. designed the study, performed the tests, collected data, and prepared the draft. D.D. and S.B. provided support during data analysis. S.S.D. wrote the manuscript. Each author reviewed the manuscript before submission.

\section{Conflict of Interest}

The authors declare that they have no conflict of interest.

\section{Acknowledgment}

We are thankful to all the staffs of department of transfusion medicine for their support.

\section{References}

1 Brilakis ES, Patel VG, Banerjee S. Medical management after coronary stent implantation: a review. JAMA 2013;310(2): 189-198

2 Nijjer SS, Watson G, Athanasiou T, Malik IS. Safety of clopidogrel being continued until the time of coronary artery bypass grafting in patients with acute coronary syndrome: a meta-analysis of 34 studies. Eur Heart J 2011;32(23):2970-2988

3 Despotis GJ, Filos KS, Zoys TN. Hogue CW Jr, Spitznagel E, Lappas DG. Factors associated with excessive postoperative blood loss and hemostatic transfusion requirements: a multivariate analysis in cardiac surgical patients. Anesth Analg 1996;82(1):13-21

4 Fitchett D, Eikelboom J, Fremes S, et al. Dual antiplatelet therapy in patients requiring urgent coronary artery bypass grafting surgery: a position statement of the Canadian Cardiovascular Society. Can J Cardiol 2009;25(12):683-689

5 Ferraris VA, Brown JR, Despotis GJ, et al; Society of Thoracic Surgeons Blood Conservation Guideline Task Force; Society of Cardiovascular Anesthesiologists Special Task Force on Blood Transfusion; International Consortium for Evidence Based Perfusion. 2011 update to the Society of Thoracic Surgeons and the Society of Cardiovascular Anesthesiologists blood conservation clinical practice guidelines. Ann Thorac Surg 2011;91(3):944-982

6 Weitzel NS, Weitzel LB, Epperson LE, Karimpour-Ford A, Tran ZV, Seres T. Platelet mapping as part of modified thromboelastography $(T E G \circledast)$ in patients undergoing cardiac surgery and cardiopulmonary bypass. Anaesthesia 2012;67(10):1158-1165

7 Estcout LJ, Birchall J, Allard S, et al. Guidelines for use of platelets: British Society of Hematology. Br J Haematol 2017; 176:365-394

8 TEG 5000 Haemostasis Analyser: User manual. Niles, IL: Haemoscope Corporation; 2008

9 Bhardwaj V, Kapoor PM, Hasija S, Singh U. Simulation in thromboelastography: platelet mapping assay (Part - I) Ann Card Anaesth 2016;19(3):530-532

10 Datta SS, De D. The impact of thromboelastography on blood transfusion policy in adult cardiac surgery-a retrospective observational study from Eastern India. Indian J Hematol Blood Transfus 2020;10.1007/s12288-020-01310-y

11 Islam AM, Patel PM. Preventing serious sequelae after an acute coronary syndrome: the consequences of thrombosis versus bleeding with antiplatelet therapy. J Cardiovasc Pharmacol 2010;55(6):585-594

12 Collyer TC, Gray DJ, Sandhu R, Berridge J, Lyons G. Assessment of platelet inhibition secondary to clopidogrel and aspirin therapy in preoperative acute surgical patients measured by 
thrombelastography platelet mapping. Br J Anaesth 2009; 102(4):492-498

13 Preisman S, Kogan A, Itzkovsky K, Leikin G, Raanani E. Modified thromboelastography evaluation of platelet dysfunction in patients undergoing coronary artery surgery. Eur J Cardiothorac Surg 2010;37(6):1367-1374

14 Breet NJ, van Werkum JW, Bouman HJ, et al. Comparison of platelet function tests in predicting clinical outcome in patients undergoing coronary stent implantation. JAMA 2010; 303(8):754-762

15 Tantry US, Bliden KP, Gurbel PA. Overestimation of platelet aspirin resistance detection by thrombelastograph platelet mapping and validation by conventional aggregometry using arachidonic acid stimulation. J Am Coll Cardiol 2005; 46(9):1705-1709

16 Bochsen L, Wiinberg B, Kjelgaard-Hansen M, Steinbrüchel DA, Johansson PI. Evaluation of the TEG platelet mapping assay in blood donors. Thromb J 2007;5:3

17 Kwak YL, Kim JC, Choi YS, Yoo KJ, Song Y, Shim JK. Clopidogrel responsiveness regardless of the discontinuation date predicts increased blood loss and transfusion requirement after off-pump coronary artery bypass graft surgery. J Am Coll Cardiol 2010;56(24):1994-2002

18 Mahla E, Suarez TA, Bliden KP, et al. Platelet function measurement-based strategy to reduce bleeding and waiting time in clopidogrel-treated patients undergoing coronary artery bypass graft surgery: the timing based on platelet function strategy to reduce clopidogrel-associated bleeding related to CABG (TARGET-CABG) study. Circ Cardiovasc Interv 2012;5(2):261-269

19 Charif F, Hamdan R, Youness G, et al. Dual antiplatelet therapy up to the time of non-elective coronary artery bypass grafting with prophylactic platelet transfusion: is it safe? J Cardiothorac Surg 2019;14(1):202

20 Karanjkar A, Kapoor PM, Sharan S, et al. A prospective randomized clinical trial of efficacy of algorithm-based point of care guided hemostatic therapy in cyanotic congenital heart disease surgical patients. J Card Crit Care 2020;3:8-16 